antibacterial component (for the treatment of purulent inflammation), as well as cerumenolytics for cleaning of the external auditory meatus and the prevention of external otitis and conductive hearing loss. Within 6 months of observation a positive tendency was noted.

The child was referred to a geneticist. Mutation was detected in exon 2 of the EDA gene - c.466C> $\mathrm{T}$ in a homozygous state and the diagnosis of hypohydrotic ectoderm dysplasia was confirmed. Vaccination against Str.

Pneumoniae and $\mathrm{H}$. influenzae type $\mathrm{b}$ was recommended for the prevention of frequent respiratory infections.

Conclusions The treatment of children with hypohydrotic ectoderm dysplasia is complex and includes thorough skin and mucous membrane care, vaccination to prevent respiratory infections and development of complications. For early diagnosis and the correct treatment of patients, the awareness of pediatricians and other specialists of the symptoms of rare hereditary diseases as well as interaction with geneticists are of utmost importance.

\section{DIAGNOSTIC DILEMMA: VACCINATION SITE REACTION OR INJECTION-RELATED CELLULITIS?}

Beatriz Vieira*, Ana Rita Curval, Juliana da Silva Cardoso, Gracinda Oliveira. Centro Hospitalar da Póvoa de Varzim/Vila do Conde, Póvoa de Varzim, Portugal

10.1136/archdischild-2021-europaediatrics.33

A previously healthy five-year-old boy presented to the paediatric emergency department with marked swelling, redness and warmth in the left arm (Figure 1), that occurred within 48 hours of receiving the fifth booster dose of diphtheria-tetanusacellular pertussis-inactivated poliovirus vaccine (DTPa-IPV). $\mathrm{He}$ was haemodynamically stable, afebrile and had a good overall appearance. His upper limb function was unaffected.

Given the clinical similarities between this large inflammatory injection site reaction (ISR) and an injection-related bacterial cellulitis, we opted, in this case, for prescribing one-week course of antimicrobial therapy with oral flucloxacillin $(50 \mathrm{mg} /$ $\mathrm{kg}$ every $8 \mathrm{~h}$ ). He showed a marked improvement within 3 days and had a complete resolution of all inflammatory signs in the left arm at one-week follow-up (Figure 2).

DTPa-IPV vaccine can cause large local inflammatory reactions (including pain, itching, swelling or redness around the site of injection), especially after the fifth (preschool) dose. Approximately 20\% will experience a minor and common ISR and approximately $2 \%$ will experience a severe ISR2, (extensive limb swelling from shoulder to elbow). Particularly large reactions can be confused with bacterial cellulitis, which is extremely uncommon, and antibiotics may be unnecessarily prescribed.

The exact pathogenesis to this reactions is uncertain but they seem to result from a cellular immunity to vaccine antigens. In general they begin a few hours following vaccination, peak at 24 to 48 hours and resolve spontaneously within a week without sequelae. Systemic symptoms, including fever, are infrequent.

Symptomatic management is recommended including analgesia and cool compress.

Antibiotic treatment or the use of anti-inflammatory medication does not reduce the duration and severity of such reactions. Moving the limb is also recommended because will encourage lymphatic drainage and prevent joint stiffness.

This case serves as a reminder that severe ISR, although infrequent, is well-described after DTPa-IPV containing vaccines. So it is important that parents of children who receive this preschool booster dose are informed of this risk and how to manage it. It is also important to explain that a history of a severe ISR is not a contraindication to future vaccines.

In conclusion, if a child presents with an extensive limb swelling after vaccination, the presumptive diagnosis must be of ISR, unless there are systemic signs (fever and toxicity) or the situation gets worse. Careful consideration is necessary to avoid missing a diagnosis of bacterial cellulitis, which although vanishingly rare, requires an appropriate antibiotic treatment at an early stage.

\section{OTITIS MEDIA WITH EFFUSION IN EXTREMELY PREMATURE CHILDREN}

VP Novikova*, IV Savenko, ES Garbaruk, MY Boboshko. Saint Petersburg State Pediatric Medical University, Russia; Pavlov First Saint Petersburg State Medical University, Russia

\subsection{6/archdischild-2021-europaediatrics.34}

Extremely preterm children (born under 28 weeks) have a high risk for hearing loss, one of the reasons for which is otitis media with effusion (OME). The higher prevalence of OME in preterm born children relative to term ones is explained by the morphofunctional immaturity, organs' pathologies, adverse side effects of the treatment received in the NICU. The aim of study was to assess the prevalence of OME, its duration in extremely preterm children and to analyze the risk factors of OME in this population.

109 extremely preterm born children from 6 months to 15 years old were observed prospectively. The mean gestation age was $26.7 \pm 1.3$ weeks; the mean birth weight was $971 \pm 197 \mathrm{~g}$. All children underwent ENT assessment with otomicroscopy and audiological evaluation at least twice during the first year of their life and at least once a year for children older than 12 months. The diagnosis of chronic OME was established with disease duration of more than 8 weeks; the diagnosis of recurrent OME was confirmed in case of the presence at least two recurrences of the disease within 18 months.

64 children $(58.7 \%)$ were identified with OME, $70.3 \%$ of them with bilateral effusion. OME was revealed in 47 children (43.1\%) during their first year of life, the disease was more common in the second half-year. Chronic OME was established in $54.7 \%$ of all children. In addition to wellknown risk factors for OME a high association of OME with perinatal infections and bronchopulmonary dysplasia (BPD) was noted in extremely preterm children. In the first year of life OME was associated with perinatal infections in $63.8 \%$ of children, and BPD in $68.1 \%$. Incidence of recurrent $\mathrm{OME}$ was significantly higher in children with BPD $(p<0.05)$.

In the study OME was shown to be a common, recurring disorder in extremely preterm children. It can lead to hearing problems causing speech, language, cognitive, and academic delay. One of the causes of OME was infections in the perinatal period. Children with BPD had a higher risk of OME and an increased risk of chronic/recurrent OME. The high incidence of OME in extremely premature children, especially 
given OME's asympotomatic presentation, requires long-term ENT and audiological monitoring at least until the age of 3 years.

\section{LUNG SEQUESTRATION IN PATIENT WITH TRANSPOSITION OF THE GREAT ARTERIES (D-TGA) - WHY IS IMPORTANT TO DIAGNOSE THIS LUNG ANOMALY}

Sanja Dorner*, Ivan Pavić, Borna Biljan, Igor Nikolić. University Hospital Osijek, Pediatric Clinic, Medical Faculty Osijek

\subsection{6/archdischild-2021-europaediatrics.35}

In this case we present a patient who was hospitalized in our hospital at the age of 3 hours due to progressive respiratory failure and cyanosis. The birth occurred spontaneously at 35 weeks of gestation, BM $3020 \mathrm{~g}$, BL $50 \mathrm{~cm}$, Apgar 3,7. Immediately after birth neonate was tachidispnoic and generally cyanotic. Despite application of surfactant and mechanical ventilation, oxygen saturation does not improve. Echocardiography revealed transposition of the great arteries (d-TGA). After a balloon atrioseptostomy, a short-term improvement occurs, but soon the right-sided pneumothorax develops and after its resolution patient was sent to a foreign cardiac surgery center where a complete cardiac surgery correction of TGA was successfully made. The postoperative course was complicated by sinus thrombosis and echocardiography shows an excellent postoperative finding.

The patient continues treatment at a local hospital which was closer to his residence and where was retreated almost every 2 months for the next 4 years because of recurrent bronchoconstriction and right-sided pneumonia.

Extensive allergologically immune diagnostic treatment has been made due to suspected immunodeficiency and allergic lung disease. During such an episode of recurrent right-sided pneumonia, as there was no improvement in treatment, patient was transferred to our facility. Immediately upon arrival, a CT scan of the chest was performed to detect the existence of an intralobar pulmonary sequester in the upper and posterobasal segment of the lower lung lobe. A thoracotomy was performed and the lung sequester was surgically removed, without complications. Since then, our patient is healthy, has not had a single respiratory infection in the last 2 years, which has significantly improved his quality of life. Lung sequestration is a rare congenital lung anomaly that belongs to the group of congenital cystic malformations with an incidence of $0.15 \%-6.4 \%$. There is two forms of sequestration - intralobar and extralobar. Symptoms of the disease are often atypical and disease is often unrecognized, according to literature data, in $71 \%$ of cases, which significantly affects on success of treatment and the quality of life of these patients. Therefore, it is necessary for physicians, especially pediatricians, to raise awareness of this disorder so it can be diagnosed and adequately cured. Some authors even recommend surgical treatment before the onset of the problem as soon as it is diagnosed, to avoid the possible complications, such as hemothorax, fungal and TB infection, malignant tissue alteration in the sequester.

\section{SERUM CALPROTECTIN IS A NOVEL PREDICTOR OF BACTERIAL URINARY TRACT INFECTION IN A FEBRILE CHILD}

Mirta Lamot*, Marijana Miler, Nora Nikolac Gabaj, Ivana Trutin, Renata Vrsalović, Iva Mihatov Štefanović, Biserka Čičak, Inga Sitaš, Mandica Vidović, Lovro Lamot, Slaven Abdović, Miroslav Harjaček. Sestre milosrdnice University Hospital Center

\subsection{6/archdischild-2021-europaediatrics.36}

Although self-limiting viral infections are the principal cause of fever in the majority of cases, it is estimated that $5 \%$ of febrile children younger than three years of age would have a urinary tract infection (UTI). Despite the increased availability of laboratory testing and development of various clinical scores, there is still no reliable predictor of UTI in children.

Recent advancements in the understanding of inflammatory process have highlighted the role of S100 proteins, with many studies suggesting their clinical value as a biomarker of inflammation. This research aims to investigate the possible role of serum calprotectin (sCAL), a heterodimer consisting of S100A8 and S100A9 proteins from S100 family, as a biomarker for bacterial urinary tract infection in children.

Patients aged 0-36 months who came to Pediatric Emergency Department of Sestre milosrdnice University Hospital Center with fever $\geq 38^{\circ} \mathrm{C}$, lasting $<72 \mathrm{~h}$, positive urine leukocyte esterase and no history of chronic illness or ongoing antibiotic use, were enrolled. In every patient, sCAL was measured with Quantum Blu rapid test, along with urine culture analysis and testing of standard inflammatory markers. Patients with bacterial count of $\geq 10^{\wedge} 5 \mathrm{CFU} / \mathrm{mL}$ in urine culture were defined as having UTI, while patients with negative culture, absent signs of other bacterial infection, positive influenza, adenovirus, and/or respiratory syncytial virus test, and/or characteristic symptoms were diagnosed with viral acute respiratory infection (ARI).

Among 52 patients included in the study (median age 6 months, IQR 3,1 - 13,8), 30 were diagnosed with ARI and 22 with UTI. In patients with UTI, the median value of sCAL was 5,6 ug/mL (IQR 3,9 - 6,3), which was significantly higher $(p<0,0001)$ than in patients with ARI, whose median value of sCAL was 2,6ug/mL (IQR 1,7 - 3,5). There was a moderate positive correlation of $\mathrm{sCAL}$ with WBC $(\mathrm{r}=0,64 ; \mathrm{p}<0,01)$ and CRP $(\mathrm{r}=0,55 ; \mathrm{p}<0,01)$. The sCAL had area under curve (AUC) of 0.93 (95\% CI 0.85 - 100) for predicting UTI, and with the optimal cut-off $(3,95 \mathrm{ug} / \mathrm{mL})$, sensitivity was $93,1 \%$ (95\% CI

78,0 - 98,8\%), specificity 76,19\% (95\% CI 54,9 - 89,4\%) and positive likelihood ratio was 3,91 .

The results of this study have shown that sCAL is a good predictor of bacterial UTI in a febrile child presenting to an emergency department.

Besides, the correlation of sCAL with duration of fever and inflammatory markers standardly used to infer the presence of bacterial infection was only moderate or insignificant, suggesting the potential differences in their dynamics. Hence, the sCAL concentration in febrile children could add to timely and accurate diagnosis of UTI, which is essential to reduce treatment delay and improve treatment outcomes. 